\title{
Micheliolide alleviates ankylosing spondylitis (AS) by suppressing the activation of the NLRP3 inflammasome and maintaining the balance of Th1/Th2 via regulating the NF-KB signaling pathway
}

\author{
Zhong-Gu Tian ${ }^{1 *}$, Miaomiao $\mathrm{Yao}^{2 *}$, Jie $\mathrm{Chen}^{3 \wedge}$ \\ ${ }^{1}$ Department of Orthopedics, Pinggu Hospital, Beijing Hospital of Traditional Chinese Medicine, Beijing, China; ${ }^{2}$ Drug Clinical Trial Institution, \\ Xi'an Gaoxin Hospital, Xi'an, China; ${ }^{3}$ Department of Osteoporosis, Gansu Provincial Hospital of Traditional Chinese Medicine, Lanzhou, China \\ Contributions: (I) Conception and design: All authors; (II) Administrative support: J Chen; (III) Provision of study materials or patients: ZG Tian, \\ M Yao; (IV) Collection and assembly of data: ZG Tian, M Yao; (V) Data analysis and interpretation: ZG Tian, M Yao; (VI) Manuscript writing: All \\ authors; (VII) Final approval of manuscript: All authors. \\ \#These authors contributed equally to this work. \\ Correspondence to: Jie Chen. Department of Osteoporosis, Gansu Provincial Hospital of Traditional Chinese Medicine, Lanzhou, China. \\ Email: chenjielz@163.com.
}

Background: Ankylosing spondylitis (AS) is a common form of inflammatory arthritis. Micheliolide (MCL), a sesquiterpene lactone, is reportedly involved in the alleviation of inflammatory response. This study aimed to investigate the mechanism of MCL in the treatment of AS.

Methods: Mice were randomly divided into five groups: the sham group, the MCL (50 mg/ $\mathrm{kg})$ group, the AS model group, the AS + MCL (20 mg/kg) group, and the AS + MCL (50 mg/ $\mathrm{kg}$ ) group. After the addition of the inhibitor celastrol, mice were randomly divided into five groups: the sham group, the AS model group, the AS + MCL $(50 \mathrm{mg} / \mathrm{kg})$ group, the AS + Celastrol $(1 \mathrm{mg} / \mathrm{kg})$ group, and the AS + Celastrol $(1 \mathrm{mg} / \mathrm{kg})+$ MCL $(50 \mathrm{mg} / \mathrm{kg})$ group.

Results: Compared with the AS model mice, the protein expression levels of tumor necrosis factor- $\alpha$ (TNF- $\alpha$ ), interleukin (IL)-1 $\beta$, IL-6, and IL-18 were decreased after MCL treatment. The protein expression levels of capase-1 p10, IL-1 $\beta$ p17, NOD-like receptor family and pyrin domain containing 3 (NLRP3), caspase-1, and apoptosis-associated speck-like protein (ASC) were also reduced. The protein expression levels of Interferon (IFN)- $\gamma$ were down-regulated, but levels of IL-4 were increased. Western blotting and immunohistochemistry revealed that the levels of $\mathrm{p}-\mathrm{I} \kappa \mathrm{B} \alpha$ were up-regulated, while the levels of phosphorylated-p65 were down-regulated. After the addition of celastrol, MCL treatment significantly reduced the levels of p-p65, NLRP3, caspase-1, and ASC. Meanwhile, the levels of IFN- $\gamma$ were markedly down-regulated, but the levels of IL-4 were enhanced.

Conclusions: Our study found that MCL suppressed the activation of NLRP3 inflammasome and maintained the balance of Th1/Th2 via regulating NF- $\mathrm{KB}$ signaling. Therefore, MCL could potentially be used to treat AS.

Keywords: Micheliolide; ankylosing spondylitis (AS); NOD-like receptor family and pyrin domain containing 3 (NLRP3); Th1/Th2; nuclear factor-kappa B (NF-кB)

Submitted Jun 04, 2020. Accepted for publication Jul 29, 2020.

doi: 10.21037/atm-20-4987

View this article at: http://dx.doi.org/10.21037/atm-20-4987

\footnotetext{
$\wedge$ ORCID: 0000-0003-3943-9195.
} 


\section{Introduction}

Ankylosing spondylitis (AS) is a chronic inflammatory rheumatic disease. Patients with AS often experience sacroiliitis and inflammatory changes of the axial skeleton, and develop syndesmophytes and bony ankylosis of the spine (1). One study has showed that the prevalence of AS in the United States increases from $0.04 \%$ to $0.09 \%$ between 2006 and 2016, with the mean age of patients range from 49.52 to 50.00 years old in the same period. Between 2006 and 2016, the proportion of AS patients who are women rose from $40 \%$ to over $47 \%$ (2). A variety of genetic and environmental factors are linked to the pathogenesis of AS (3). Moreover, the body's ability to negatively regulate immune response is impaired with AS, and this might also be related to its etiology (4).

Micheliolide (MCL), a guaianolide sesquiterpene lactone, is derived from Michelia compressa and Michelia champaca and can also be in vitro synthesized from parthenolide (5). Previous research has shown that MCL exerts an inhibitive effect on lipopolysaccharide (LPS)-induced inflammatory response and can protect mice from LPS challenge through the nuclear factor-kappa B (NF- $\mathrm{BB})$ and phosphoinositol 3 kinase $(\mathrm{PI} 3 \mathrm{~K}) /$ protein kinase B (AKT) pathways (6). Research has also demonstrated that in mycobacterium tuberculosis, MCL plays an anti-inflammatory role by downregulating NLRP3 inflammasome activation via the PI3K/ Akt/NF-kB pathway (7). Moreover, MCL alleviates hepatic steatosis through the inhibition of inflammation and promotion of autophagy (8). Furthermore, MCL inhibits $\mathrm{NF}-\kappa \mathrm{B}$ activation, and attenuates carcinogenesis in AOM/ DSS-induced colorectal cancer (CRC) (9). Based on these encouraging findings, we speculated that MCL might play a protective role in AS in mice and subsequently designed this study to test our hypothesis. Therefore, this study aimed to investigate the mechanism of MCL in the treatment of AS.

We present the following article in accordance with the ARRIVE reporting checklist (available at http://dx.doi. org/10.21037/atm-20-4987).

\section{Methods}

\section{AS model}

All animal experiments were performed in accordance with the NIH Guide for the Care and Use of Laboratory Animals and received approval from the Ethics Committee of Beijing Hospital of Traditional Chinese Medicine. Specific-pathogen-free (SPF) BALB/c mice aged 5-
6 weeks old, were obtained from the Beijing Weitonglihua Experimental Animal Technology Co., Ltd. The mice were housed in a controlled environment at $25 \pm 3{ }^{\circ} \mathrm{C}$, humidity $60 \%$, under a 12-h light/dark cycle with free access to water. Mice were randomly divided into five groups: the sham group, the MCL $(50 \mathrm{mg} / \mathrm{kg}$ ) group, the AS model group, the AS + MCL $(20 \mathrm{mg} / \mathrm{kg})$ group, and the AS + MCL $(50 \mathrm{mg} / \mathrm{kg})$ group. After the addition of the inhibitor celastrol, mice were randomly divided into five groups: the sham group, the AS model group, the AS + MCL (50 mg/ $\mathrm{kg}$ ) group, the AS + Celastrol $(1 \mathrm{mg} / \mathrm{kg})$ group, and the AS + Celastrol $(1 \mathrm{mg} / \mathrm{kg})+$ MCL $(50 \mathrm{mg} / \mathrm{kg})$ group.

Proteoglycan lyophilized powder was dissolved in $1 \times$ phosphate buffer saline (PBS) $(\mathrm{pH}=7.4)$ to a concentration of $10 \mathrm{mg} / \mathrm{mL}$ on ice. Dimethyloctadecyl ammonium bromide (DDA) was selected as the immunoadjuvant and was dissolved in $1 \times \mathrm{PBS}(\mathrm{pH}=7.4)$ to a concentration is $20 \mathrm{mg} / \mathrm{mL}$ on ice. Then, $100 \mu \mathrm{L}$ of proteoglycan solution and DDA solution was mixed well. Female BALB/c mice were intraperitoneally injected with $200 \mu \mathrm{L}$ of mixed solution (10) to establish the AS mouse model. An equivalent volume of PBS was administered to the model and normal control mice.

MCL was supplied by Accendatech Co., Ltd. (Tianjin, China) and was prepared daily by being fully dissolved in normal saline solution. The model and normal control group mice were given an equivalent volume of saline solution each day. Each group of mice was treated for 16 weeks. The mice were given daily injections of Celastrolat a dose of $1 \mathrm{mg} / \mathrm{kg}(11)$.

\section{Cytokine enzyme-linked immunosorbent assay (ELISA)}

Samples of peripheral blood were collected from the AS model mice and the serum was separated. The TNF- $\alpha$, IL-6, IL-1 $\beta$, IL-18, IFN- $\gamma$, and IL-4 levels in the serum were detected, according to the protocol of the manufacturer. A Bio-Rad Microplate reader (CA, USA) was used to determine the optical density, and absorption was measured at $450 \mathrm{~nm}$. A cytokine standard supplied with the ELISA Kit was used to establish a standard curve for each measurement.

\section{Flow cytometry analysis}

Peripheral blood mononuclear cells (PBMCs) were collected from the AS mice for flow cytometry. The PBMCs were added to a tube containing antibody and dyeliquor, and 
incubated for $15 \mathrm{~min}$ in darkness. Then, $2 \mathrm{~mL} 1 \times$ Lysing Solution was added and the samples were incubated for a further $30 \mathrm{~min}$ in darkness, before centrifugation at 1,500 rap and removal of the supernatant. Subsequently, $2 \mathrm{~mL}$ PBS was added, and the samples were centrifuged at 1,500 rap and the supernatant was removed. Finally, $500 \mu \mathrm{L}$ PBS was added. Analysis of the samples was performed with a FACS Aria cell sorter (BD Biosciences), and FlowJo (Tree Star, OR) was used to analyze the data.

\section{Western blot}

PBMCs were lysed with $200 \mu \mathrm{L}$ of radioimmunoprecipitation assay (RIPA) buffer (Thermo Scientific, USA) containing protease inhibitor (Roche, Germany). Then, $50 \mu \mathrm{g}$ of total protein was loaded into $10 \%$ sodium dodecyl sulfate-polyacrylamide gel electrophoresis (SDS-PAGE) loading buffer, and the proteins were transferred to polyvinylidene difluoride (PVDF) membranes. After sealing with $5 \%$ skimmed milk at $37^{\circ} \mathrm{C}$ for $120 \mathrm{~min}$, the PVDF membranes were incubated at $4{ }^{\circ} \mathrm{C}$ overnight with the primary antibodies against capase-1 p10 (Abcam, 1:1,000), IL-1 $\beta$ p17 (R\&D Systems, Minneapolis, MN, USA; 1:1,000), NLRP3 (Cell Signaling, 1:1,000), caspase-1 (Cell Signaling, 1:1,000), ASC (Cell Signaling, 1:1,000), p-p65 (Cell Signaling, 1:1,000), and p-IкB $\alpha$ (Cell Signaling, 1:1,000) or Lamin-B (Cell Signaling, 1:1,000). Following that, the membranes were incubated with goat anti-rabbit IgG horseradish peroxidase (HRP)-conjugated secondary antibodies at $37{ }^{\circ} \mathrm{C}$ for $60 \mathrm{~min}$. An automatic digital gel image analysis system Bio-Rad CFX-96 (Bio-Rad, CA, USA) was employed to determine and analyze the band densities.

\section{Immunobistochemistry}

PBMCs were incubated overnight with primary antibodies against p65 (Cell Signaling, 1:800). The corresponding second antibodies were incubated at room temperature for 1 h. An Olympus DX51 fluorescence microscope (Olympus, Tokyo, Japan) was used to observe the cells. Data analysis was performed with Image 6.0 (Media Cybernetics, USA).

\section{Statistical analyses}

All experiments were independent and were carried out at least in triplicate. Experimental data were presented as the mean \pm standard deviation (SD). Statistical analyses were performed using SPSS 22.0 software (SPSS Inc., Chicago, IL, USA). Student's $t$-test was used to compare the groups. Differences between groups were compared using oneway analysis of variance (ANOVA), and Duncan's method was used as a post-hoc test. $\mathrm{P}<0.05$ was considered to be statistically significant, and differences were considered significant at $\mathrm{P}<0.05$.

\section{Results}

MCL alleviated the levels of TNF- $\alpha, I L-1 \beta, I L-6$, and IL-18 in the AS model

Previous research has indicated that the levels of TNF- $\alpha$, IL-1 $\beta$, and IL-6 are distinctly up-regulated in AS patients, making them attractive therapeutic targets (12). The result of ELISA revealed that the protein expressive levels of TNF- $\alpha$, IL-1 $\beta$, IL- 6 , and IL- 18 were increased in the AS model mice compared to the sham group $(\mathrm{P}<0.05)$ (Figure 1). Following MCL treatment, the levels of TNF- $\alpha$, IL- $1 \beta$, IL-6, and IL-18 were decreased compared with the AS model mice, especially in the high concentration group $(\mathrm{P}<0.05)$. These findings suggested that MCL had an alleviative effect on inflammatory response in the AS model.

\section{MCL alleviated the levels of Caspase1 p10, IL1及 p17, NLRP3, caspase-1, and ASC in the AS model}

One previous study has reported the possible involvement of the NLRP3 inflammasome in the pathogenesis of AS, which means it can potentially be a therapeutic target in AS treatment (1). In accordance with these results, we found that the protein expression levels of caspase-1 p10, IL1 $\beta$ p17, NLRP3, caspase-1, and ASC in the AS model mice were notably higher than in the sham group mice (Figure 2) $(\mathrm{P}<0.05)$. However, the levels of caspase-1 p10, IL1 $\beta$ p17, NLRP3, caspase-1, and ASC were decreased after MCL treatment $(\mathrm{P}<0.05)$. The protein expression levels of caspase1 p10, IL1 $\beta$ p17, NLRP3, caspase-1, and ASC were not significantly different between the MCL $(50 \mathrm{mg} / \mathrm{kg})$ group and the sham group (Figure 2) $(\mathrm{P}>0.05)$. These results suggested that MCL could suppress the activation of the NLRP3 inflammasome in the AS model.

\section{MCL down-regulated IFN- $\gamma$ levels and up-regulated IL-4 levels in AS model}

Imbalance in the levels of Th1 and Th2-secreted cytokines 

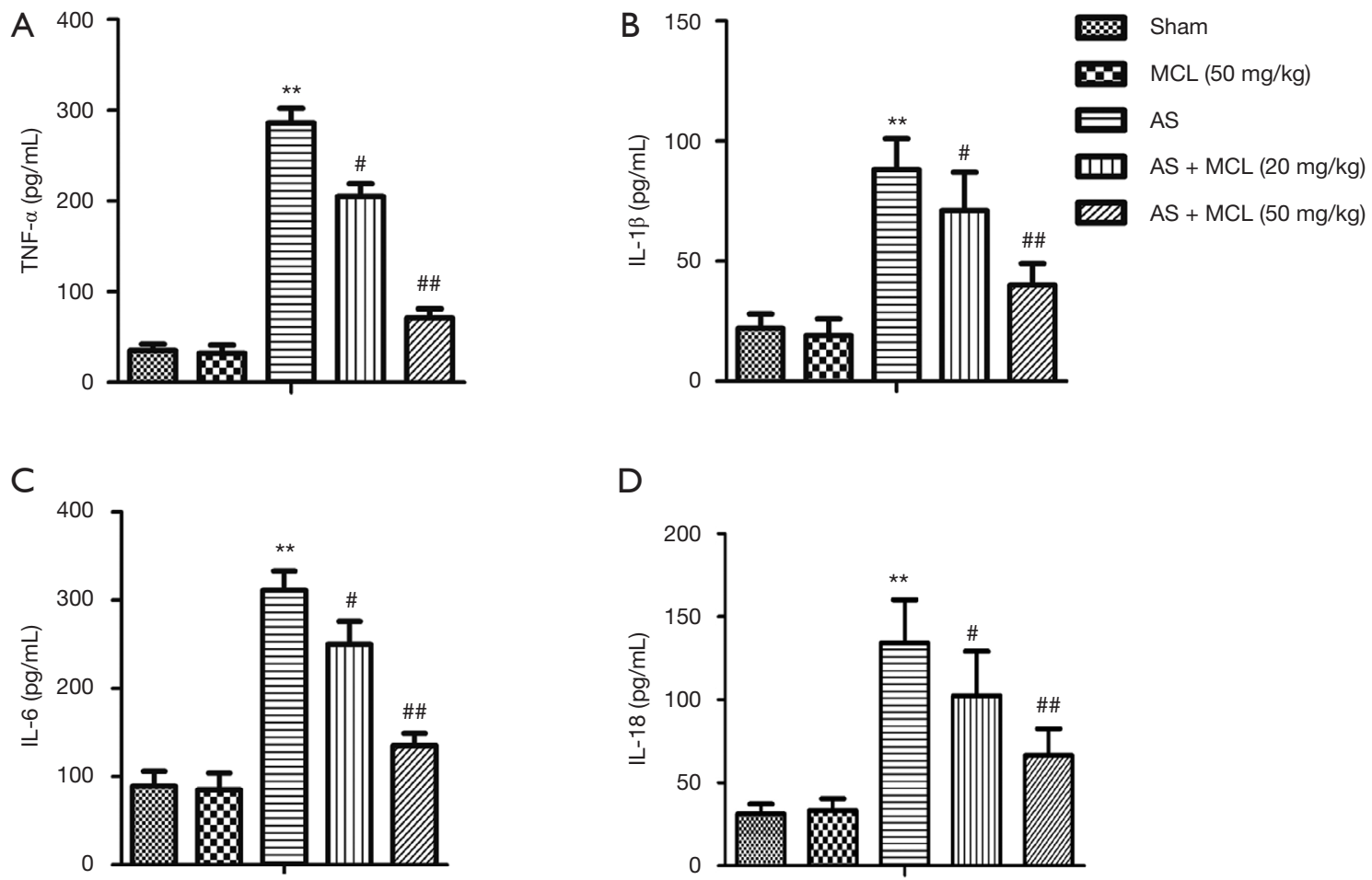

Figure $1 \mathrm{MCL}$ alleviated the levels of TNF- $\alpha, \mathrm{IL}-1 \beta, \mathrm{IL}-6$, and IL-18 in the AS model. The protein expression levels of TNF- $\alpha$, IL-1 $\beta$, IL6, and IL-18 were detected by ELISA. (A) TNF- $\alpha$ (pg/mL), (B) IL-1 $\beta$ (pg/mL), (C) IL-6 (pg/mL), (D) IL-18 (pg/mL). The results are presented as mean $\pm \mathrm{SD}$ and represent three individual experiments. ${ }^{* *}, \mathrm{P}<0.01$ versus sham group; ${ }^{\#}, \mathrm{P}<0.05 ;$;, $\mathrm{P}<0.01$ versus AS model group.

may be critical to the development of autoimmune diseases (13). Remarkably, elevated levels of Th1 cytokines and reduced levels of Th2 cytokines in the peripheral blood of patients with AS can indicate disease activity (14). The levels of IFN- $\gamma$ and IL-4 were detected by ELISA and flow cytometry, respectively. As shown Figure 3, the result of ELISA demonstrated that the levels of IFN- $\gamma$ were markedly enhanced in AS model group compared with the sham group $(\mathrm{P}<0.05)$. Meanwhile, the levels of IL-4 were markedly inhibited $(\mathrm{P}<0.05)$. However, the levels of IFN- $\gamma$ were significantly down-regulated after treatment with MCL in contrast with that in the AS model group, while the levels of IL-4 were markedly up-regulated $(\mathrm{P}<0.05)$. The result of flow cytometry was consistent with the findings of ELISA. These results suggested that MCL can adjust the balance of Th1/Th2 in the AS model.

\section{MCL down-regulated p-p65 levels and up-regulated $p$-IK $B$ a levels in AS model}

In mammals, the NF- $\mathrm{BB}$ signaling pathway mainly involves

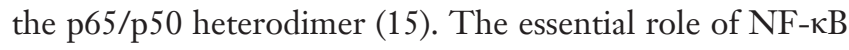
signaling in the progression of AS, rheumatoid arthritis, and different cancers has been described previously (16-18). As Figure 4 shows, the protein levels of p-p65 were increased, while the levels $\mathrm{p}-\mathrm{I} \kappa \mathrm{B} \alpha$ were decreased in AS model group compared with those sham group. After treatment with MCL, the levels of p-p65 were decreased $(\mathrm{P}<0.05)$, but the levels of $\mathrm{p}-\mathrm{I} \kappa \mathrm{B} \alpha$ were increased $(\mathrm{P}<0.05)$. At the same time, the result of immunohistochemistry showed that the percentage of p65-positive rate also were notably lower after MCL treatment $(\mathrm{P}<0.05)$. These findings indicated that MCL might play a protective role in mice with AS via regulating the NF- $\mathrm{KB}$ signaling pathway.

\section{MCL alleviated activation of the NLRP3 inflammasome and maintained the balance of $T b 1 / T h 2$ via regulating the $N F-\kappa B$ signaling pathway}

To further demonstrate whether MCL played a role in regulating the NF- $\kappa \mathrm{B}$ signaling pathway in the AS, we added celastrol to inhibit the NF- $\kappa B$ pathway and explore 

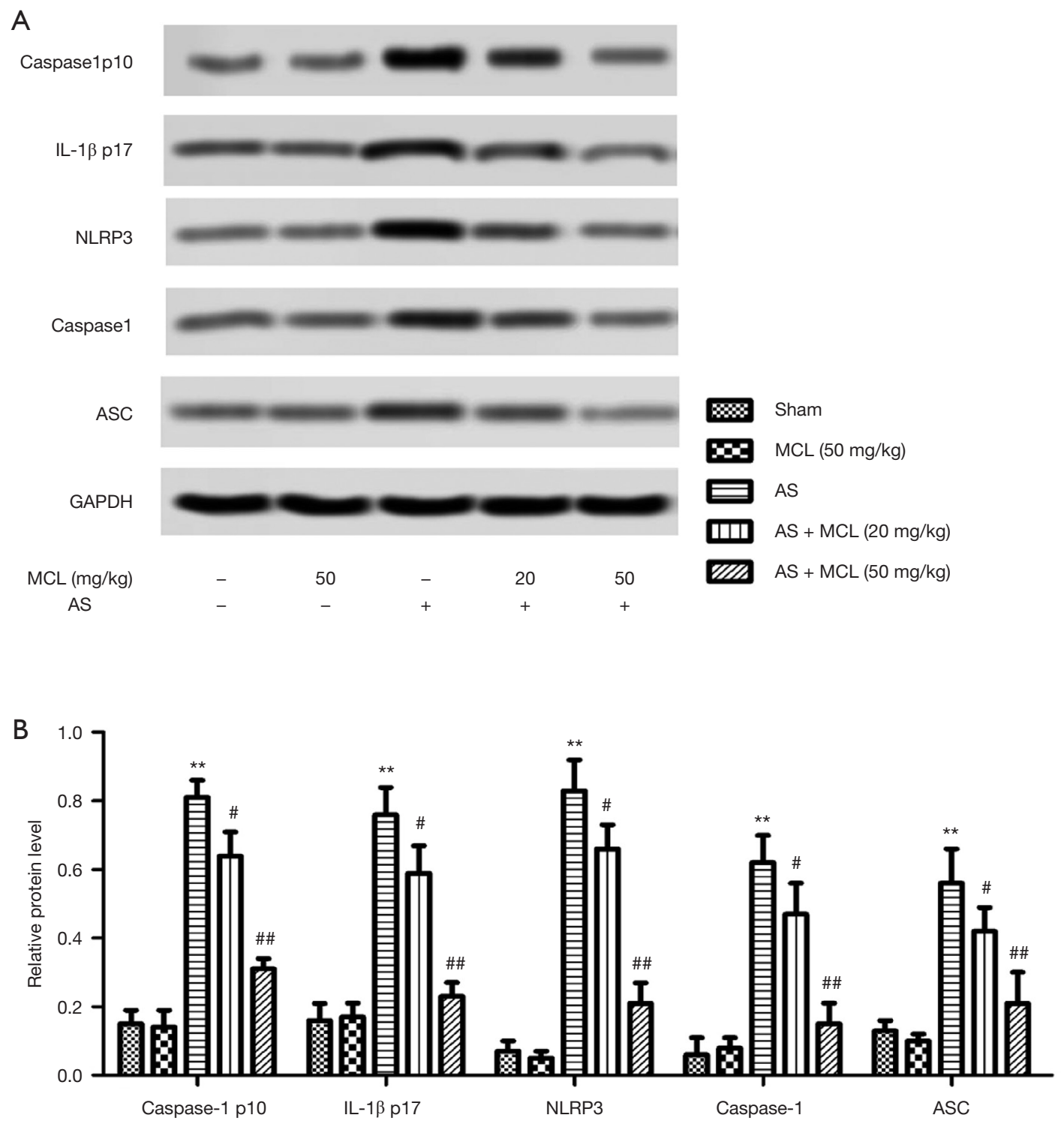

Figure 2 MCL alleviated the levels of caspase-1 p10, IL-1 $\beta$ p17, NLRP3, caspase-1, and ASC in the AS model. (A)The protein expression levels of caspase-1 p10, IL1 $\beta$ p17, NLRP3, caspase-3, and ASC were detected by Western blot. (B) Semi-quantitative analysis of the relative levels of caspase-1 p10, IL1 $\beta$ p17, NLRP3, caspase-3, and ASC in each group of mice was performed. The results are presented as mean \pm $\mathrm{SD}$ and represent three individual experiments. ${ }^{* *}, \mathrm{P}<0.01$ versus sham group; ${ }^{\#}, \mathrm{P}<0.05 ;{ }^{\#}, \mathrm{P}<0.01$ versus AS model group.

the function of MCL in AS model mice. Celastrol, an NF$\kappa \mathrm{B}$ inhibitor, was found to ameliorate hypercalciuria and articular cartilage lesions in a secondary osteoporosis mouse model (11). As shown in Figure 5, the protein expression levels of p-p65, NLRP3, caspase-1, and ASC were lower in the group with inhibitor than in the AS model mice $(\mathrm{P}<0.05)$. Interestingly, following intervention with MCL, the protein expression levels of p-p65, NLRP3, caspase-1, and ASC were lower than in the group with inhibitor $(\mathrm{P}<0.05)$. At the same time, the levels of IFN- $\gamma$ in the inhibitor group were significantly repressed in contrast with those in the AS model group, while the levels of IL-4 were markedly elevated $(\mathrm{P}<0.05)$. Furthermore, after intervention with MCL, the levels of IFN- $\gamma$ were more significantly repressed compared with the inhibitor group, while the levels of IL-4 were more markedly elevated. These results suggested that MCL could alleviate AS through suppressing the activation of the NLRP3 inflammasome and maintaining the balance 

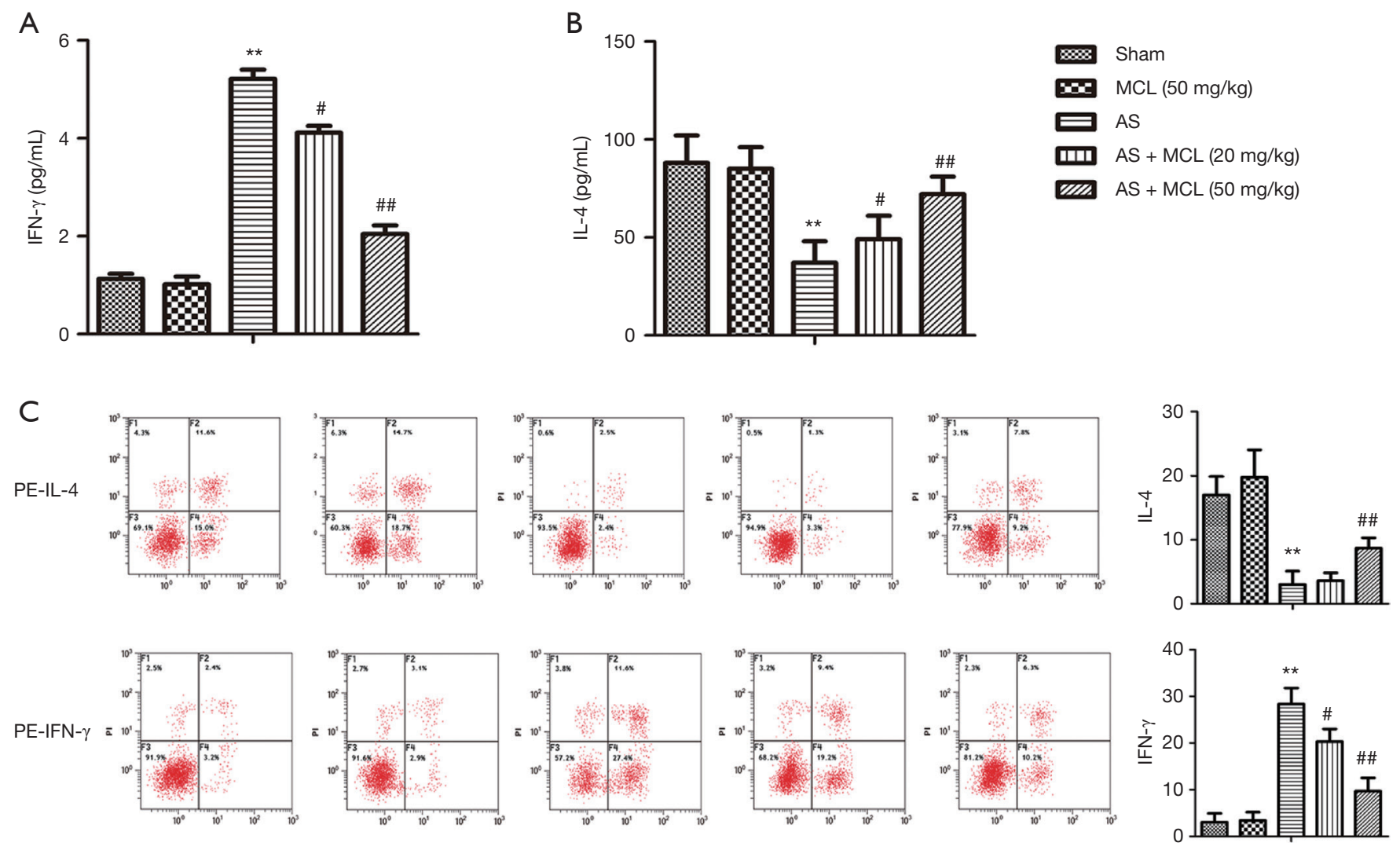

Figure 3 MCL down-regulated IFN- $\gamma$ levels and up-regulated IL-4 levels in the AS model. The protein expression levels of IFN- $\gamma$ and IL-4 were detected by ELISA and flow cytometry, respectively. (A) IFN- $\gamma$ (pg/mL), (B) IL-4 (pg/mL), (C) representative flow cytometry plots for IL-4 and IFN- $\gamma$. The results are presented as mean $\pm \mathrm{SD}$ and represent three individual experiments. **, $\mathrm{P}<0.01$ versus sham group; , $\mathrm{P}<0.05,{ }^{\#}, \mathrm{P}<0.01$ versus AS model group.

of Th1/Th2 via the regulation of the NF- $\mathrm{BB}$ signaling pathway in our AS model.

\section{Discussion}

Ankylosing spondylitis (AS) is a common form of inflammatory arthritis. Wang et al. (19) has found that the levels of IL-23 and IL-17 are elevated in serum collected from patients with active AS. Further, IL-17 has been shown to enhance T-cell activation and stimulate a variety of cells (fibroblasts, endothelial and epithelial cells, and macrophages) to produce proinflammatory mediators [interleukin-1 $\beta$ (IL-1 $\beta$ ), IL-6, TNF- $\alpha$, and chemokines] (20). Previous research has indicated that TNF- $\alpha$, IL-1 $\beta$, and IL-6 are distinctly up-regulated in AS patients (12). More importantly, MCL reduces the secretion of IL- 6 , TNF- $\alpha$, IL-1 $\beta, M C P-1$, IFN- $\beta$ and IL-10 in sera, and ameliorates lung and liver damage (6). In this study, the AS model mice showed the expression levels of TNF- $\alpha$, IL-1 $\beta$, and IL-6 were significantly elevated. Meanwhile, we found the expressions levels of TNF- $\alpha$, IL-1 $\beta$, IL-6, and IL-18 were decreased in the AS model mice after intervention with MCL.

NOD-like receptor family and pyrin domain containing 3 (NLRP3) inflammasome activity is central to the development of various inflammatory diseases. Both adapter protein ASC and pro-caspase- 1 are activated by NLRP3 inflammasome, which leads to caspase-1 activation. Subsequently, interleukin-1 $\beta$ (IL-1 $\beta$ ), IL-18, or high mobility group box-1 protein (HMGB1) are secreted to exert a regulating effect $(21,22)$. Research has indicated that NLRP3 inflammasome is associated with susceptibility to AS (23). Interestingly, MCL plays an important role in modulating Mycobacterium tuberculosis (Mtb)-induced 
A

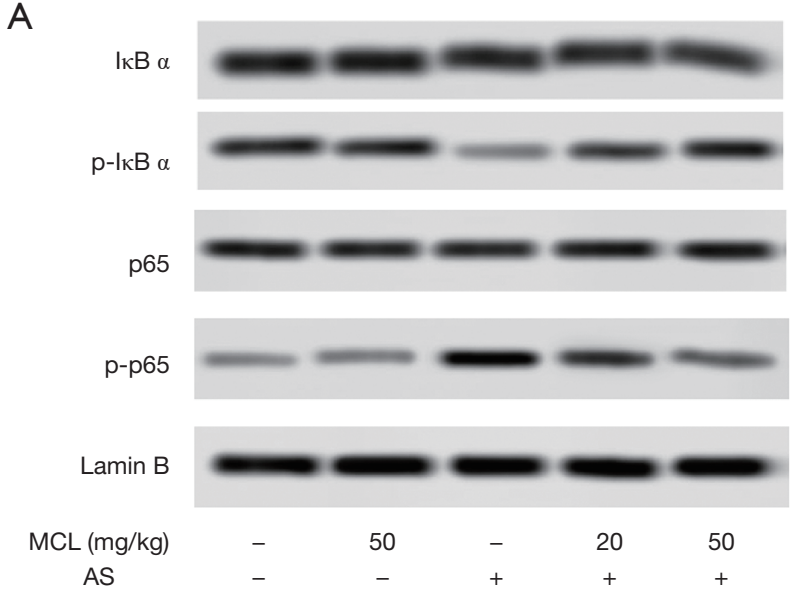

B

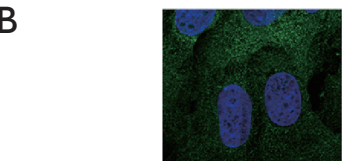

$\mathrm{MCL}(\mathrm{mg} / \mathrm{kg})$

AS

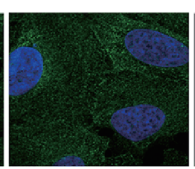

50

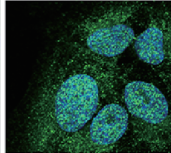

$-$

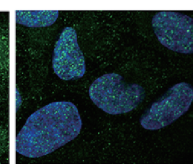

20

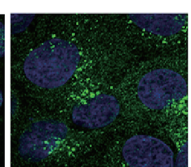

50
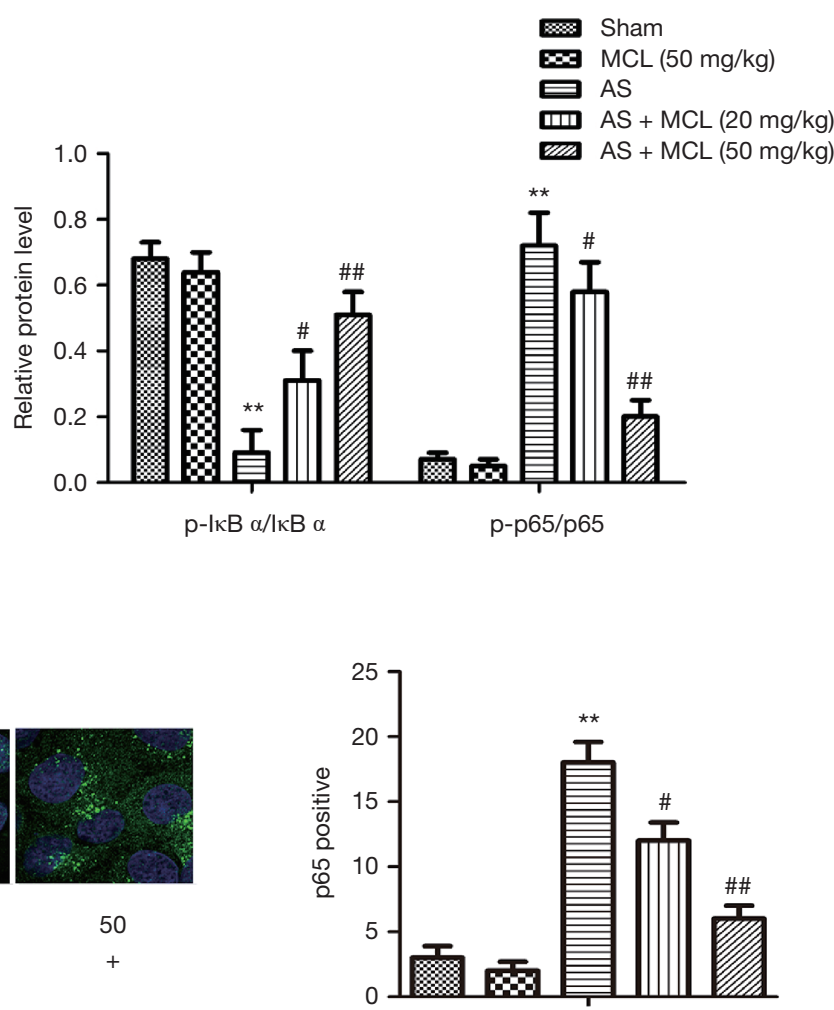

Figure 4 MCL down-regulated the level of p-p65 and up-regulated that of p-IкB $\alpha$ in the AS model. (A)The protein expression levels of p-p65 and $\mathrm{p}-\mathrm{I} \kappa \mathrm{B} \alpha$ were detected by Western blot. Semi-quantitative analysis of the relative levels of p-p65 and p-IкB $\alpha$ in each group of mice was performed. (B) The protein expression of p65 was detected by immunohistochemical staining. Magnification 200×. Semi-quantitative analysis of the relative amounts of $\mathrm{p} 65$ in each group of mice was performed. The results are presented as mean \pm SD and represent three individual experiments. **, $\mathrm{P}<0.01$ versus sham group; ${ }^{\#}, \mathrm{P}<0.05 ;{ }^{\# \#}, \mathrm{P}<0.01$ versus $\mathrm{AS}$ model group.

inflammatory response through down-regulating the activation of NLRP3 inflammasome (7). The result of this article showed that the levels of capase- 1 p10, IL-1 $\beta$ p17, NLRP3, caspase-1, and ASC in AS model mice were notably higher. Remarkably, the levels of capase-1 p10, IL-1 $\beta$ p17, NLRP3, caspase-1, and ASC were decreased following MCL treatment. These results suggested that MCL could alleviate AS by suppressing the activation of the NLRP3 inflammasome.

CD4+ T cells, and their subsets includingTh1, Th2 and Th17, regulatory $\mathrm{T}$ cells, follicular helper T cells, and Th9, have multiple functions (24). Interferon- $\gamma(\mathrm{IFN}-\gamma)$ is known to be a Th1 cytokine and is the only type II interferon. Recently, abnormal expression of IFN- $\gamma$ has been reported to be associated with a variety of auto-inflammatory and immune diseases $(25,26)$. The Th1-secreted cytokine IFN- $\gamma$ antagonizes the secretion of Th2 cytokines IL- 4 and IL-10, thereby inhibiting Th2 response (27). Previous research has showed that the expression profile of IFN- $\gamma$ has an obvious association with rs2430561 polymorphism, suggesting that rs2430561 minor allele may regulate the expression of IFN- $\gamma$, thus contributing to AS risk (28). According to previous research, IL-4 attenuates the pathogenesis of AS via modulating macrophage polarization (29). This study revealed that the levels of IFN- $\gamma$ were markedly enhanced, while the levels of IL-4 were markedly inhibited. However, the levels of IFN- $\gamma$ were suppressed whereas the levels of IL-4 were increased following MCL treatment. In methicillin-resistant S. aureus (MRSA) infection mouse model, MCL down-regulated the expression of IL-6, TNF- $\alpha$, MCP-1/CCL2 and IFN- $\gamma$ in sera, and ameliorated the organ damage of liver and kidney (30). These results suggested that MCL could adjust the balance of Th1/Th2.

$\mathrm{NF}-\kappa \mathrm{B}$, a ubiquitous family of transcription factors, is involved in a variety of essential processes in the body including inflammation, immunity, and cell proliferation 

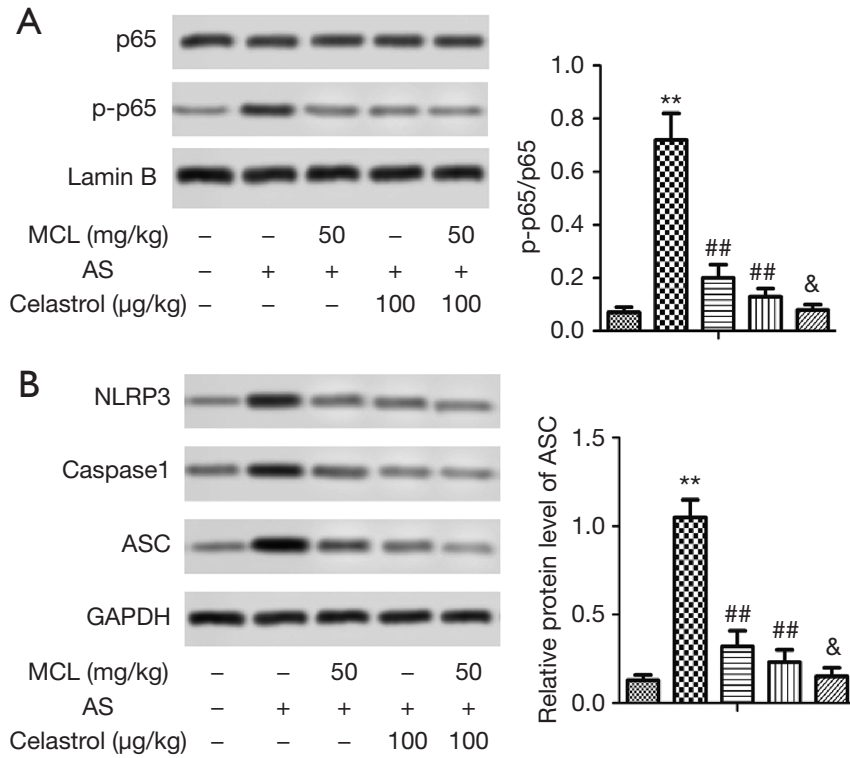
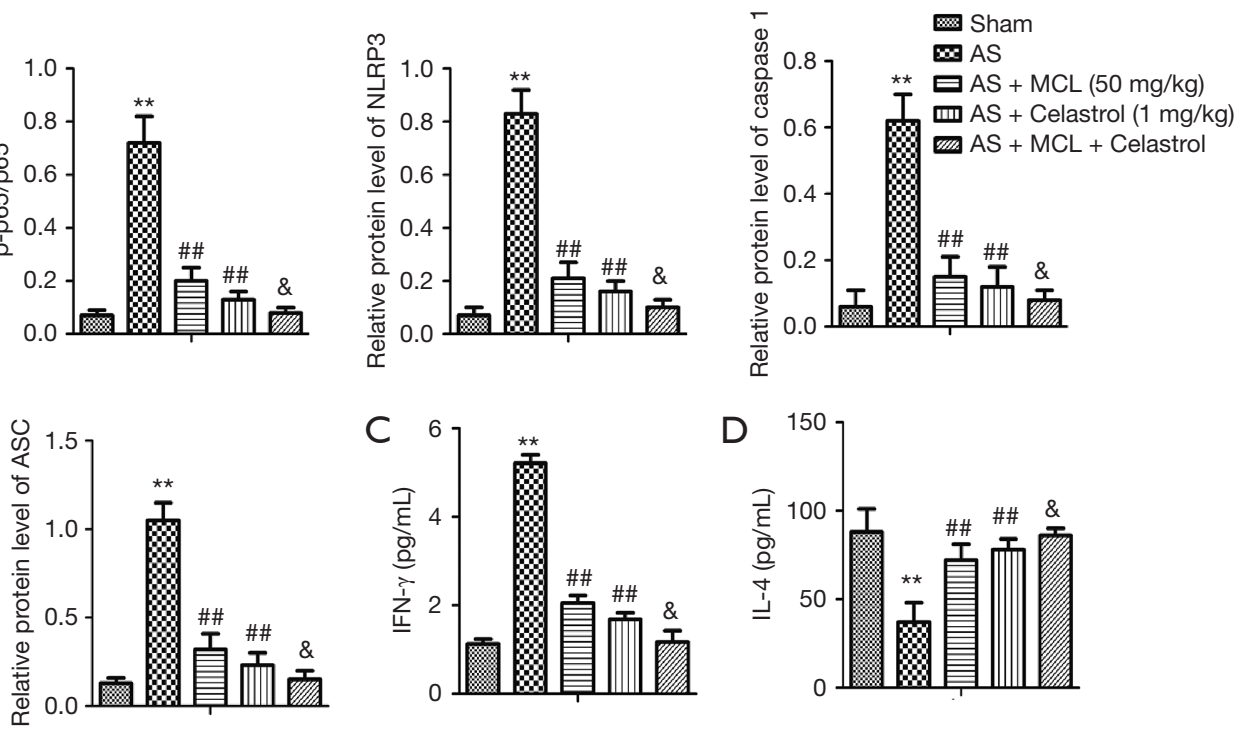

Figure $5 \mathrm{MCL}$ alleviated activation of the NLRP3 inflammasome and maintained the balance of Th1/Th2 via regulating the NF- $\mathrm{BB}$ signaling pathway. (A) A representative result of Western blot analysis. Semi-quantitative analysis of the protein expression levels of p65 phosphorylation from the sham group, AS model group, AS + MCL $(50 \mathrm{mg} / \mathrm{kg})$ group, AS + Celastrol (1 mg/ $\mathrm{kg})$ group, and AS + celastrol (1 mg/kg) + MCL (50 mg/kg) group. (B) A representative result of Western blot analysis of the protein expression levels of NLRP3, caspase-3, and ASC. Semi-quantitative analysis of the relative level of NLRP3, caspase-3, and ASC in each group of mice was performed. The protein expression levels of IFN- $\gamma$ and IL-4 were detected by ELISA. (C) IFN- $\gamma$ (pg/mL). (D) IL-4 (pg/mL). The results are presented as mean $\pm \mathrm{SD}$ and represent three individual experiments. ${ }^{* *}, \mathrm{P}<0.01$ versus Sham group; ${ }^{\# \#}, \mathrm{P}<0.01$ versus AS model group; ${ }^{*}, \mathrm{P}<0.05$ versus $\mathrm{AS}+$ celastrol $(1 \mathrm{mg} / \mathrm{kg})$ group.

and survival (31). AS is a common autoimmune disease, and its pathogenesis is closely related to immune function (32). The genetically determines high activity of the tumor necrosis factor- $\alpha$ (TNF- $\alpha$ ), IL-23/IL-17, and NF- $\kappa$ B pathways increases the risk of AS (33). MCL attenuates the inflammatory responses and lipid accumulation in lipid mixture (LM)-treated AML12 and L02 cells by upregulating PPAR- $\gamma$ and decreasing $\mathrm{p}-\mathrm{I \kappa \textrm {B }} \alpha$ and $\mathrm{p}-\mathrm{NF}-\kappa \mathrm{B} /$ p65 (8). MCL suppresses LPS-stimulated the activation of $I \kappa B \alpha / N F-\kappa B$ pathway and Akt pathway (34). In our study, the levels of p-p65 were notably elevated, while the levels of $\mathrm{p}-\mathrm{I \kappa \textrm {B }} \alpha$ were decreased in the AS mouse model. Interestingly, after treatment with MCL, the levels of p-p65 were notably decreased, while the levels of p-IкB $\alpha$ were increased. These results suggested that MCL could inhibit the activation of the NF- $\kappa B$ pathway.

To further demonstrate whether MCL played a role in regulating the NF- $\kappa \mathrm{B}$ signaling pathway in the AS, we added celastrol to inhibit the NF- $\mathrm{KB}$ pathway and explore the function of MCL in AS model mice. Celastrol, an NF- $\mathrm{\kappa B}$ inhibitor, was found to ameliorate hypercalciuria and articular cartilage lesions in a secondary osteoporosis mouse model (11). Celastrol significantly inhibits cell proliferation of isolated AS fibroblasts and in vitro osteogenic differentiation (35). Curculigoside A (CA) effectively down-regulates the expression of NF-KB/NLRP3 pathway on adjuvant arthritis (AA) rats (36). Epigallocatechin gallate maintains the Th1/Th2 balance and suppresses immunoinflammatory response in colonic tissue through the TLR4/MyD88/NF- $\mathrm{BB}$ signaling pathway (37). The anti-inflammatory mechanism of citrus tachibana leaves ethanol extract (CTLE) may involve the modulation of Th1/Th2 imbalance via inhibition of NF- $\mathrm{KB}$ signaling and histamine secretion (38). In the research, after intervention with MCL, the protein expression levels of p-p65, NLRP3, caspase- 1 and ASC were lower than in the inhibitor group. The levels of IFN- $\gamma$ were more significantly repressed compared with the inhibitor group, while the levels of IL-4 were more markedly elevated. These results suggested that MCL alleviated AS via regulating the NF- $\kappa B$ signaling 
pathway.

In summary, MCL inhibited TNF- $\alpha$, IL- $1 \beta$, IL- 6 , and IL-18 expression, and reduced the protein expression levels of capase- 1 p10, IL- $1 \beta$ p17, NLRP3, caspase-3, and ASC. It also suppressed the expression of IFN- $\gamma$ but increased that of IL-4. In addition, the levels of p-p65 were decreased, while the levels of $\mathrm{p}-\mathrm{I} \kappa \mathrm{B} \alpha$ were increased. MCL alleviated AS by suppressing the activation of the NLRP3 inflammasome and maintaining the balance of Th1/Th2 via regulation of the NF- $\mathrm{kB}$ signaling pathway.

\section{Acknowledgments}

This article thanks Beijing Hospital of Traditional Chinese Medicine, Xi'an Gaoxin Hospital, and Gansu Provincial Hospital of Traditional Chinese Medicine for its support. Funding: None.

\section{Footnote}

Reporting Checklist: The authors have completed the ARRIVE reporting checklist. Available at http://dx.doi. org/10.21037/atm-20-4987

Data Sharing Statement: Available at http://dx.doi. org/10.21037/atm-20-4987

Conflicts of Interest: All authors have completed the ICMJE uniform disclosure form (available at http://dx.doi. org/10.21037/atm-20-4987). The authors have no conflicts of interest to declare.

Ethical Statement: The authors are accountable for all aspects of the work in ensuring that questions related to the accuracy or integrity of any part of the work are appropriately investigated and resolved. All animal experiments were performed in accordance with the NIH Guide for the Care and Use of Laboratory Animals and received approval from the Ethics Committee of Beijing Hospital of Traditional Chinese Medicine (approval ID: No. 20190320).

Open Access Statement: This is an Open Access article distributed in accordance with the Creative Commons Attribution-NonCommercial-NoDerivs 4.0 International License (CC BY-NC-ND 4.0), which permits the noncommercial replication and distribution of the article with the strict proviso that no changes or edits are made and the original work is properly cited (including links to both the formal publication through the relevant DOI and the license). See: https://creativecommons.org/licenses/by-nc-nd/4.0/.

\section{References}

1. Kim SK, Cho YJ, Choe JY. NLRP3 inflammasomes and NLRP3 inflammasome-derived proinflammatory cytokines in peripheral blood mononuclear cells of patients with ankylosing spondylitis. Clin Chim Acta 2018;486:269-74.

2. Walsh J, Hunter T, Schroeder K, et al. Trends in diagnostic prevalence and treatment patterns of male and female ankylosing spondylitis patients in the United States, 2006-2016. BMC Rheumatol 2019;3:39.

3. Tsui FW, Tsui HW, Akram A, et al. The genetic basis of ankylosing spondylitis: new insights into disease pathogenesis. Appl Clin Genet 2014;7:105-15.

4. Chen Z, Qi J, Wei Q, et al. Variations in gut microbial profiles in ankylosing spondylitis: disease phenotyperelated dysbiosis. Ann Transl Med 2019;7:571.

5. Zhai JD, Li D, Long J, et al. Biomimetic semisynthesis of arglabin from parthenolide. J Org Chem 2012;77:7103-7.

6. Qin X, Jiang X, Jiang X, et al. Micheliolide inhibits LPSinduced inflammatory response and protects mice from LPS challenge. Sci Rep 2016;6:23240.

7. Zhang Q, Jiang X. MCL Plays an Anti-Inflammatory Role in Mycobacterium tuberculosis-Induced Immune Response by Inhibiting NF-kappaB and NLRP3 Inflammasome Activation. Mediators Inflamm 2017;2017:2432904.

8. Zhong J, Gong W, Chen J, et al. Micheliolide alleviates hepatic steatosis in $\mathrm{db} / \mathrm{db}$ mice by inhibiting inflammation and promoting autophagy via PPAR-gamma-mediated NF-small ka, CyrillicB and AMPK/mTOR signaling. Int Immunopharmacol 2018;59:197-208.

9. Viennois E, Xiao B, Ayyadurai S, et al. Micheliolide, a new sesquiterpene lactone that inhibits intestinal inflammation and colitis-associated cancer. Lab Invest 2014;94:950-65.

10. Glant TT, Mikecz K. Proteoglycan aggrecan-induced arthritis: a murine autoimmune model of rheumatoid arthritis. Methods Mol Med 2004;102:313-38.

11. Liu X, Cai F, Zhang Y, et al. Celastrol, an NF-kappaB inhibitor, ameliorates hypercalciuria and articular cartilage lesions in a mouse model of secondary osteoporosis. J Pharmacol Sci 2016;130:204-11.

12. Tarner IH, Müller-Ladner U, Uhlemann C, et al. The 
effect of mild whole-body hyperthermia on systemic levels of TNF-alpha, IL-1beta, and IL-6 in patients with ankylosing spondylitis. Clin Rheumatol 2009;28:397-402 .

13. Bautista-Caro MB, Arroyo-Villa I, Castillo-Gallego C, et al. Decreased frequencies of circulating follicular helper $\mathrm{T}$ cell counterparts and plasmablasts in ankylosing spondylitis patients Naive for TNF blockers. PLoS One 2014;9:e107086.

14. Wen JT, Zhang DH, Fang PF, et al. Role of Th1/Th2 cytokines in the diagnosis and prognostic evaluation of ankylosing spondylitis. Genet Mol Res 2017. doi: 10.4238/ gmr16019322.

15. Taniguchi K, Karin M. NF-אB, inflammation, immunity and cancer: coming of age. Nat Rev Immunol 2018;18:309-24.

16. Fang L, Liu J, Wan L, et al. Xinfeng capsule improves hypercoagulative state by inhibiting miR-155/NF- $\mathrm{kB}$ signaling pathway in patients with active ankylosing spondylitis. Xi Bao Yu Fen Zi Mian Yi Xue Za Zhi 2016;32:1094-8.

17. Noort AR, Tak PP, Tas SW. Non-canonical NF-kB signaling in rheumatoid arthritis: Dr Jekyll and Mr Hyde? Arthritis Res Ther 2015;17:15.

18. Shostak K, Chariot A. EGFR and NF- $\kappa B$ : partners in cancer. Trends Mol Med 2015;21:385-93.

19. Wang X, Lin Z, Wei Q, et al. Expression of IL-23 and IL17 and effect of IL-23 on IL-17 production in ankylosing spondylitis. Rheumatol Int 2009;29:1343-7.

20. Jethwa $H$, Bowness P. The interleukin (IL)-23/IL-17 axis in ankylosing spondylitis: new advances and potentials for treatment. Clin Exp Immunol 2016;183:30-6.

21. Yang Q, Liu R, Yu Q, et al. Metabolic regulation of inflammasomes in inflammation. Immunology 2019;157:95-109.

22. Kamo N, Ke B, Ghaffari AA, et al. ASC/caspase-1/ $\mathrm{IL}-1$ beta signaling triggers inflammatory responses by promoting HMGB1 induction in liver ischemia/ reperfusion injury. Hepatology 2013;58:351-62.

23. Zhao S, Chen H, Wu G, et al. The association of NLRP3 and TNFRSF1A polymorphisms with risk of ankylosing spondylitis and treatment efficacy of etanercept. J Clin Lab Anal 2017;31:e22138.

24. Luckheeram RV, Zhou R, Verma AD, et al. CD4(+)T cells: differentiation and functions. Clin Dev Immunol 2012;2012:925135.

25. Nakajima A, Aoki Y, Sonobe M, et al. Relative expression and correlation of tumor necrosis factor- $\alpha$, interferon- $\gamma$, and interleukin-17 in the rheumatoid synovium. Clin Rheumatol 2016;35:1691-7.

26. Heidari Z, Mahmoudzadeh-Sagheb H, Hashemi M, et al. Association between IFN- $\gamma+874 \mathrm{~A} / \mathrm{T}$ and IFN- $\gamma \mathrm{R} 1(-611 \mathrm{~A} /$ G, $+189 \mathrm{~T} / \mathrm{G}$, and $+95 \mathrm{C} / \mathrm{T})$ Gene Polymorphisms and Chronic Periodontitis in a Sample of Iranian Population. Int J Dent 2015;2015:375359.

27. van der Weijden MA, van Denderen JC, Lems WF, et al. Etanercept Increases Bone Mineral Density in Ankylosing Spondylitis, but Does Not Prevent Vertebral Fractures: Results of a Prospective Observational Cohort Study. J Rheumatol 2016;43:758-64.

28. Xu H, Li B. Effect of Interferon- $\gamma$ Polymorphisms on Ankylosing Spondylitis: A Case-Control Study. Med Sci Monit 2017;23:4126-31.

29. Lin S, Qiu M, Chen J. IL-4 Modulates Macrophage Polarization in Ankylosing Spondylitis. Cell Physiol Biochem 2015;35:2213-22.

30. Jiang $X$, Wang $Y$, Qin $Y$, et al. Micheliolide provides protection of mice against Staphylococcus aureus and MRSA infection by down-regulating inflammatory response. Sci Rep 2017;7:41964.

31. Panday A, Inda ME, Bagam P, et al. Transcription Factor NF-kappaB: An Update on Intervention Strategies. Arch Immunol Ther Exp (Warsz) 2016;64:463-83.

32. Gönül Y, Genc A, Ahsen A, et al. The effects of IL-18BP on mRNA expression of inflammatory cytokines and apoptotic genes in renal injury induced by infrarenal aortic occlusion. J Surg Res 2016;202:33-42.

33. Sode J, Bank S. Genetically determined high activities of the TNF-alpha, IL23/IL17, and NFkB pathways were associated with increased risk of ankylosing spondylitis. BMC Med Genet 2018;19:165.

34. Sun Z, Li G, Tong T, et al. Micheliolide suppresses LPS-induced neuroinflammatory responses. PLoS One 2017;12:e0186592.

35. Zou YC, Yang XW, Yuan SG, et al. Celastrol inhibits prostaglandin E2-induced proliferation and osteogenic differentiation of fibroblasts isolated from ankylosing spondylitis hip tissues in vitro. Drug Des Devel Ther 2016;10:933-48.

36. Ding H, Gao G, Zhang L, et al. The protective effects of curculigoside A on adjuvant-induced arthritis by inhibiting NF-кB/NLRP3 activation in rats. Int Immunopharmacol 2016;30:43-9.

37. Bing X, Xuelei L, Wanwei D, et al. EGCG Maintains Th1/Th2 Balance and Mitigates Ulcerative Colitis Induced 
by Dextran Sulfate Sodium through TLR4/MyD88/NFkappaB Signaling Pathway in Rats. Can J Gastroenterol Hepatol 2017;2017:3057268.

38. Bui TT, Piao CH, Kim SM, et al. Citrus tachibana Leaves Ethanol Extract Alleviates Airway Inflammation by the

Cite this article as: Tian ZG, Yao M, Chen J. Micheliolide alleviates ankylosing spondylitis (AS) by suppressing the activation of the NLRP3 inflammasome and maintaining the balance of Th1/Th2 via regulating the NF- $\kappa \mathrm{B}$ signaling pathway. Ann Transl Med 2020;8(16):991. doi: 10.21037/atm20-4987
Modulation of Th1/Th2 Imbalance via Inhibiting NFkappaB Signaling and Histamine Secretion in a Mouse Model of Allergic Asthma. J Med Food 2017;20:676-84.

(English Language Editor: J. Reynolds) 